\title{
Polishing the crystal ball: mining multi-omics data in dermatomyositis
}

\author{
Rochelle L. Castillo ${ }^{1}$, Alisa N. Femia ${ }^{2}$ \\ ${ }^{1}$ Department of Medicine, Division of Rheumatology, NYU Grossman School of Medicine, New York, NY, USA; ${ }^{2}$ Ronald O. Perelman Department \\ of Dermatology, NYU Grossman School of Medicine, New York, NY, USA \\ Contributions: (I) Conception and design: All authors; (II) Administrative support: None; (IIII) Provision of study materials or patients: All authors; (IV) \\ Collection and assembly of articles: RL Castillo; (V) Data analysis and interpretation: All authors; (VI) Manuscript writing: All authors; (VII) Final \\ approval of manuscript: All authors. \\ Correspondence to: Alisa N. Femia, MD, Ronald O. Perelman Department of Dermatology, New York University Langone Medical Center, 240 E \\ 38th St, 11th Floor, New York, NY 10016, USA. Email: alisa.femia@nyulangone.org.
}

\begin{abstract}
Precision medicine, which recognizes and upholds the uniqueness of each individual patient and the importance of discerning these inter-individual differences on a molecular scale in order to provide truly personalized medical care, is a revolutionary approach that relies on the discovery of clinically-relevant biomarkers derived from the massive amounts of data generated by epigenomic, genomic, transcriptomic, proteomic, microbiomic, and metabolomic studies, collectively known as multi-omics. If harnessed and mined appropriately with the help of ever-evolving computational and analytic methods, the collective data from omics studies has the potential to accelerate delivery of targeted medical treatment that maximizes benefit, minimizes harm, and eliminates the "fortune-telling" inextricably linked to the prevailing trialand-error approach. For a disease such as dermatomyositis (DM), which is characterized by remarkable phenotypic heterogeneity and varying degrees of multi-organ involvement, an individualized approach that incorporates big data derived from multi-omics studies with the results of currently available serologic, histopathologic, radiologic, and electrophysiologic tests, and, most importantly, with clinical findings obtained from a thorough history and physical examination, has immense diagnostic, therapeutic, and prognostic value. In this review, we discuss omics-based research studies in DM and describe their practical applications and promising roles in guiding clinical decisions and optimizing patient outcomes.
\end{abstract}

Keywords: Dermatomyositis (DM); myositis; precision medicine; epigenomics; genomics; transcriptomics; proteomics

Submitted Jul 15, 2020. Accepted for publication Nov 10, 2020.

doi: $10.21037 /$ atm-20-5319

View this article at: http://dx.doi.org/10.21037/atm-20-5319

One of the most challenging and inescapable roles of a clinician is that of fortune-teller. One's job description has perpetually entailed reasonably predicting a patient's disease course and the most optimal treatment strategy based upon the constellation of clinical features and the current best available evidence that constitute a clinician's version of a crystal ball. While the colossal amount of information gleaned from clinical trials, descriptive studies, classification criteria, and practice guidelines is useful and indispensable, it should not stand alone in guiding decision making, as it is founded upon aggregate data extracted from an "average" group of patients and does not take into account the crucial differences inherent to each individual. These individualities leave patients with atypical presentations and/or rare diseases, such as dermatomyositis (DM), particularly vulnerable to delayed or missed diagnosis, suboptimal treatment plans, and ultimately, worse outcomes.

Recognition of this challenge led to the launching of the Precision Medicine Initiative in 2015, with the goal of "accelerating biomedical discoveries and providing clinicians with new tools, knowledge, and therapies to select which treatments will work best for which patients" $(1,2)$. 

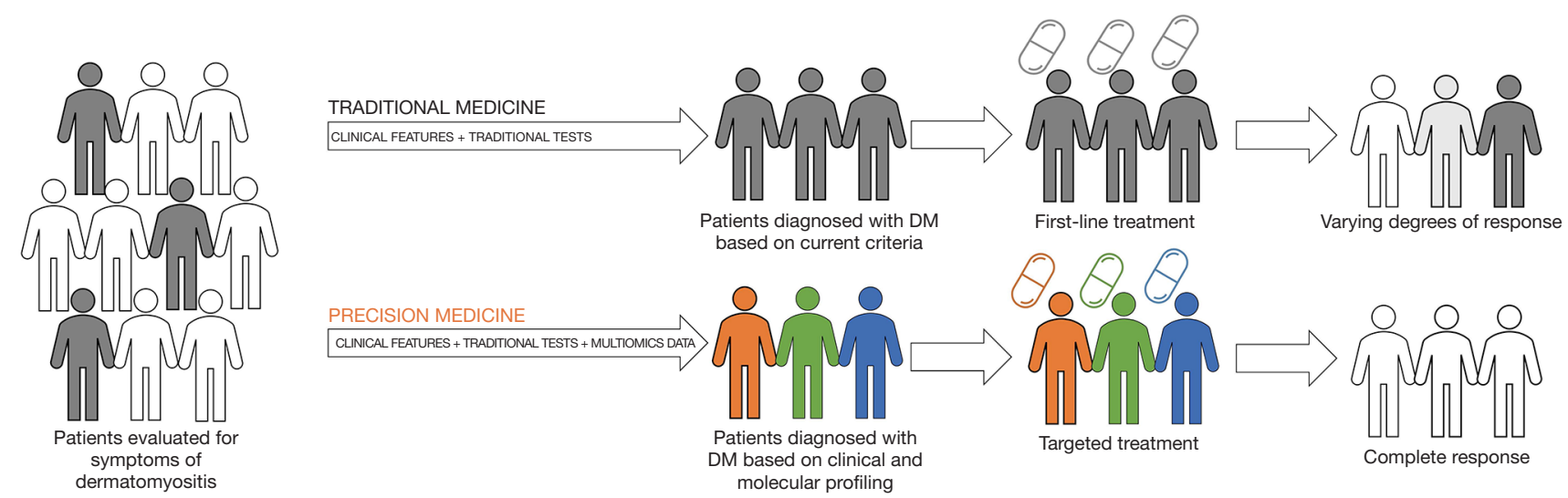

Figure 1 Paradigm of precision medicine.

Precision medicine embodies a revolutionary paradigm shift from traditional or empiric medicine in that it takes into account the uniqueness of each individual patient and the importance of discerning these inter-individual differences on a molecular scale, with the ultimate goal of developing risk stratification, disease classification, drug response prediction, drug selection, and prognostication tools that will allow clinicians to provide a truly personalized and targeted approach to patient care. To achieve this, it relies on the discovery of molecular biomarkers derived from the massive amounts of data generated by epigenomic, genomic, transcriptomic, proteomic, microbiomic, and metabolomic studies, collectively known as multi-omics or panomics, that are synthesized and converted into clinically-meaningful and actionable knowledge through computational methods such as machine learning (Figure 1) $(3,4)$.

While the transition to a patient-centric, individualized model of care would certainly be advantageous to patients across all the known disease entities, the ones who stand to benefit the most are those plagued by chronic, debilitating, poorly-understood, rare, and potentially life-threatening conditions. DM, a type of idiopathic inflammatory myopathy (IIM) characterized by a wide, continuously expanding array of cutaneous manifestations, any number of combinations of multi-organ involvement ranging from skin-limited disease to rapidly-progressive interstitial lung disease, and the strongest association with malignancy of any of the IIMs, is one such condition $(5,6)$.

Over the decades, the list of cutaneous and extracutaneous manifestations that have come to characterize DM has expanded at a rapid pace, growing beyond those manifestations included in the definition set by Peter and
Bohan in the 1970s. Consequently, a panel of DM experts from the fields of rheumatology and dermatology identified over two dozen potential criteria for defining the disease, set to be validated in a large, multi-center prospective study (7). While this, as well as other ongoing efforts such as the discovery of myositis-specific and myositis-associated antibodies, have aided tremendously in clarifying the myriad of DM phenotypes and providing guidance to clinicians, much still remains unknown as far as understanding the pathogenesis, risk factors, susceptibilities, disease definitions, and treatment response predictors. "Omic" studies hope to bridge these gaps. While we are behind the strides achieved in the field of oncology, these worthwhile efforts to advance the practice of precision medicine in rheumatologydermatology in general and dermatomyositis in particular represent significant forward motion in our understanding of autoimmune connective tissue disease. In this review, we will discuss several of these studies within the context of their potential clinical implications.

\section{Role in stratifying genetic risk}

A complex interplay between genetic and environmental risk factors and immune and non-immune mechanisms is responsible for the development of IIM in genetically susceptible individuals (8-10). Thus, genomic studies to identify high-risk single nucleotide polymorphisms (SNPs) that are associated with a particular clinical and/or autoantibody phenotype in DM patients have the potential to facilitate earlier diagnosis and predict extracutaneous organ involvement. Given that genetic variations can also influence response to and toxicity from 
medications, pharmacogenomic studies that can leverage this information to tailor drug selection and prevent unnecessary exposure to ineffective, potentially harmful treatments are on the forefront of the precision medicine movement (11), although in dermatomyositis the translation of genetic studies to the clinical therapeutic realm is yet to be established. A search of the literature failed to yield studies on pharmacogenomics in DM patients; however, a number of genomic studies that aimed to elucidate genetic susceptibility to DM and to autoantibody positivity have been performed.

Current evidence suggests that the strongest genetic risk for disease susceptibility in dermatomyositis as well as in other IIMs lies within the human leukocyte antigen (HLA) region of the major histocompatibility complex (MHC) $(9,12,13)$. Genome wide association studies (GWAS) have identified that SNPs within the HLA 8.1 ancestral haplotype, defined by HLA-A1, -B8, -Cw7, -DRB1*03:01, -DQA1*05:01, and -DQB1*02:01, are responsible for the primary genetic risk for all the clinical IIM phenotypes as well as several autoantibody phenotypes $(9,12,14)$. A large, multi-country GWAS on Caucasian patients with European ancestry revealed that HLA-DRB 1*03:01 conferred the strongest individual allelic associations for DM and JDM. Several SNPs of the HLA-DRB1*03:01 allele reached the genome-wide level of significance threshold, namely rs114042950 (and rs114388793, which is in strong LD with rs114042950) $\left(\mathrm{P}=3.9 \times 10^{-11}, \mathrm{OR}=1.51\right)$. HLA-B ${ }^{*} 08: 01$, in turn, showed the strongest association for polymyositis (PM) and anti-Jo-1 positivity (12). A follow-up study on the same cohort described significant associations between the 8.1 ancestral haplotype and autoantibody profile, specifically antiJo-1 (HLA-B ${ }^{*} 08: 01, \mathrm{P}=2.28 \times 10^{-53}$ and HLA-DRB1*03:01, $\mathrm{P}=3.25 \times 10^{-9}$ ), anti-PM/Scl (HLA-DQB $1 * 02: 01$, $\left.\mathrm{p}=1.47 \times 10^{-26}\right)$ and anti-cytosolic $5^{\prime}$-nucleotidase

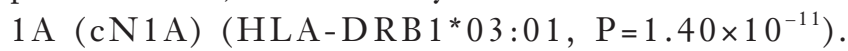
Associations independent of this haplotype were found with anti-Mi-2 (HLA-DRB1 $\left.{ }^{*} 07: 01, \mathrm{P}=4.92 \times 10^{-13}\right)$ and anti-hydroxy-3-methylglutaryl-CoA reductase (HMGCR) (HLA-DRB 1* $\left.11, \mathrm{P}=5.09 \times 10^{-6}\right)$. HLA-DQB1*02:02 was found to confer susceptibility to adult-onset transcription intermediary factor 1 gamma (TIF-1- $\gamma)\left(\mathrm{P}=2.96 \times 10^{-5}\right.$, OR $=3.31)$ and juvenile-onset TIF-1- $\gamma\left(\mathrm{P}=3.70 \times 10^{-5}, \mathrm{OR}=2.47\right)$ (15). Earlier studies on North American Caucasians with northwestern European ancestry identified the DQA1*0301 allele as a possible risk factor both DM and PM while the HLA- A*68 alleles were significant only for DM $\left(\mathrm{P}_{\text {corrected }}=0.0021\right)(16)$. In the same cohort, they also found significant associations between 8.1 alleles and myositis autoantibodies, specifically HLA-Cw*0304 and anti-PL-7 antibody $\left(\mathrm{P}_{\text {corr }}=0.046\right)$, HLA-B ${ }^{*} 5001$ and DQA1*0104 and anti-signal recognition particle (SRP) autoantibody $\left(\mathrm{P}_{\text {corr }}=0.024\right.$ and $\mathrm{P}=0.010$, respectively), and HLA DRB1*11 and DQA1*06 with anti-Ku $\left(\mathrm{P}_{\text {corr }}=0.041\right)$ and anti-La $\left(\mathrm{P}_{\text {corr }}=0.023\right)$, respectively. Another study on Caucasians revealed that HLA-DPB $1{ }^{*} 0101$ was associated with IIM overall $\left(\mathrm{P}_{\text {corr }}=2 \times 10^{-3} ; \mathrm{OR}=2\right]$ and anti-Jo- $1\left(\mathrm{P}_{\text {corr }}=3 \times 10^{-5}\right.$; OR $=4.1)(17)$.

Studies on non-Caucasian cohorts yielded ethnic differences. African American patients with IIM were not found to have strong disease associations with HLA alleles of the 8.1 ancestral haplotype; however, those with DM or with anti-Jo- 1 shared the risk factor HLA-DRB1*0301 with European Americans $\left(\mathrm{P}_{\text {corr }}=0.001, \mathrm{OR}=6.7\right)$. Novel HLA risk factors, however, were discovered in African Americans for myositis overlap (DRB1*08), for anti-SRP autoantibody (DQA1*0102) and for anti-Mi-2 autoantibody (DRB1*0302) (14). A study on Mesoamerican Mestizos did not find HLA risk factors for IIM but found that the HLADRB1*04 and DQA1*03 alleles were associated with antiMi-2 autoantibodies $(14,18)$. In a Japanese cohort, HLADRB $1 * 0803$ was associated with IIM $(\mathrm{P}=0.02, \mathrm{OR}=1.9)$ and with anti-anti-aminoacyl-tRNA synthetase (ARS) autoantibodies $(\mathrm{P}=0.04, \mathrm{OR}=2.3)$ while $\mathrm{DRB} 1{ }^{*} 0405$ was associated only with anti-ARS autoantibodies $(\mathrm{P}=0.04$, $\mathrm{OR}=2.1$ ) (19). Finally, in Chinese Han patients with DM, positive associations were found with HLA-DQA1*0104 $(\mathrm{P}=0.01, \mathrm{OR}=2.58)$ and HLA-DRB $1{ }^{*} 07(\mathrm{P}=0.01$, OR $=2.26$ ). HLA-DRB $1 * 03$, on the other hand, appeared to be protective against $\mathrm{DM}(\mathrm{P}=0.01 ; \mathrm{OR}=0.26)(20)$.

While the strongest genetic risk associations were found in genes within the MHC, other risk loci in nonMHC regions have also been discovered with varying strengths of linkage disequilibrium with HLA alleles. In the largest genetic study to date in IIM on Caucasian patients from 14 countries, a SNP in the GSDMB gene (rs1008723) was found to be exclusively associated with $\mathrm{DM}$ and JDM compared to the other IIMs $(\mathrm{OR}=1.2$, $\left.\mathrm{P}=9.05 \times 10^{-6}\right)$. The same study also found other regions (UBE2L3, CD28, TRAF6, STAT4) that were associated with IIMs as a group (and not DM or JDM specifically) (13). A study on a North American cohort of DM patients found an increased frequency in tumor necrosis factor alpha (TNF $\alpha$ )-308A polymorphism in the entire DM cohort compared to controls $(\mathrm{P}=0.003)$ that remained significant when only Caucasian patients were analyzed $(\mathrm{P}=0.014)(21)$. 
The role of the -308A polymorphism was echoed in a study on a United Kingdom-based Caucasian cohort, which revealed a strong association between the TNF-308A allele with each of the IIM subgroups $(\mathrm{OR}=2.5)$. However, this was not independent of HLA-B*08. Another TNF allele was also found to be significantly associated with $\mathrm{DM}(\mathrm{OR}$ $=2.2)$ and positive anti-synthetase $(\mathrm{OR}=2.9)$ and anti-PM$\mathrm{Scl}(\mathrm{OR}=5.6)$ antibodies (22).

Studies on other non-Caucasian cohorts likewise reported non-HLA risk susceptibility loci. In a Japanese cohort, a SNP of WDFY4, rs7919656, was found to be significantly associated with clinically amyopathic dermatomyositis $(\mathrm{CADM})\left(\mathrm{P}=1.5 \times 10^{-8}\right.$, OR $\left.=3.87\right)(23)$. Another group from Japan reported an association between C8orf13-BLK rs13277113A that, while significant for both $\mathrm{DM}$ and $\mathrm{PM}$ (when analyzed separately and combined), was strongest for $\mathrm{DM}(\mathrm{P}<0.001, \mathrm{OR}=1.64)$ compared to $\mathrm{PM}(\mathrm{P}=0.011$, OR 1.32) (24). In Chinese Han patients, a SNP of the BANK1 gene, rs3733197, was associated with a genetic predisposition to developing DM and PM $\left(\mathrm{P}_{\text {corr }}=0.026\right)$ and two SNPs (rs7117932 and rs6590330) in the ETS1 gene region were also found to be associated with $\mathrm{DM}\left(\mathrm{P}=8.79 \times 10^{-3}, \mathrm{OR}=1.25\right.$ and $\mathrm{P}=0.30,95 \%$, OR $=1.20$, respectively) $(25,26)$. A strong allele association was likewise noted between two SNPS in the TNFAIP region and IIM, namely rs2230926 ( $\left.\mathrm{P}_{\text {corr }}=7.5 \times 10^{-3}, \mathrm{OR}=1.61\right)$ and rs5029939 $\left(\mathrm{P}_{\text {corr }}=6 \times 10^{-3}, \mathrm{OR}=1.64\right)(27)$. Another study on Chinese Han patients found two phospholipase C-like 1 (PLCL1) SNPs (rs6738825 and rs7572733) to be associated specifically with DM and not with $\mathrm{PM}\left(\mathrm{P}_{\text {corr }}=0.015\right.$; $\mathrm{P}_{\text {corr }}=0.025$ in DM, respectively) (28). Finally, in a cohort from Mexico, CTN3 R577X polymorphism was found to be a genetic risk factor for $\mathrm{DM}(\mathrm{OR} 1.2, \mathrm{P}<0.001)(29)$.

\section{Role in differentiating between IIM subgroups and other DM mimics}

Although not currently utilized in the typical clinical setting, molecular profiling can also be used to differentiate between IIM subgroups and from other causes of myopathy, leading to improved diagnostic accuracy and more prompt institution of the appropriate treatment. Multiple studies have shown distinct genetic differences between the subgroups of IIMs, knowledge of which could be useful in differentiating among them in the relatively rare but real instances of clinical ambiguity (13).

In the largest genetic study to date on IIM on Caucasian patients, the magnitude of the association of PTPN22 for IIM rivaled that of HLA, reaching genome-wide level of significance $\left(\mathrm{P}<5 \times 10^{-8}\right)$. However, when adjusted for subgroups, it was not found to be significantly associated with $\mathrm{DM}(\mathrm{P}=0.19)$, and $\mathrm{PM}$ appeared to be the primary driver of this association (13). A separate study on Caucasian patients found the DRB $1{ }^{*} 07-\mathrm{DQA} 1{ }^{*} 02$ DQB1*02 haplotype to be differentially associated between $\mathrm{PM}$ and $\mathrm{DM}\left(\mathrm{OR}=0.3, \mathrm{P}_{\text {corr }}=0.002\right)$ (30). In the realm of transcriptomics, a study comparing the $\mathrm{T}$ cell transcriptome profile of peripheral blood mononuclear cells (PBMC) from DM and PM patients using bulk RNA sequencing showed over 170 differentially expressed genes in CD8+ T-cells, with 44 genes expressed higher in PM and 132 in DM patients [false discovery rate (FDR) <0.05] (31). A microarray studies on muscle biopsy specimens showed significant upregulation of FAM208B, OSBPL8, OSTM1, NIPBL, SHQ1, CDK17 and INTS6 in DM compared to PM patients (32). Thus, developing a marker set of the most subgroup-specific SNPs with the highest odds ratios and/or top differentially expressed genes based on FDR-corrected $\mathrm{P}$ values or $\log 2$ fold changes could potentially be used as a differentiation tool.

In the same manner, a genetic, transcriptomic, or proteomic panel can also help differentiate IIMs from other disease entities with similar clinical features. In one study, muscle biopsies in children with JDM were found to have significantly increased expression of type 1 interferon inducible genes compared to those with Duchenne muscular dystrophy and to healthy controls $(33,34)$. Finally, through single cell RNA sequencing of skin biopsies, the investigators developed a five-gene panel consisting of the top five differentially upregulated genes in DM, specifically IL18, LCE2D, LCE1B, KRT80, and TPM4, that was able to reliably distinguish DM from cutaneous lupus erythematosus (CLE), with an area under the curve (AUROC) of 0.98 for subacute CLE and 0.98 for discoid LE (35). Given that the lesions of DM and CLE can be clinically similar and are often histopathologically indistinguishable, this finding is helpful in discriminating between the two conditions.

\section{Role in predicting extracutaneous disease}

While the presence of myositis-specific antibodies and their correlation with distinct clinical phenotypes has been of unparalleled value in prognosticating future symptom development and organ involvement, it is not infallible given the lack of methodological standardization and the 
existence of seronegative patients, as well as the variability in clinical presentation between even those patients with the same antibody profile (36). Further, while there is substantial evidence that autoantibodies can develop years prior to the onset of symptoms in patients with systemic lupus erythematous, Sjogren's syndrome, systemic sclerosis, and rheumatoid arthritis, the precise temporal relationship between autoantibody formation and onset of clinically apparent features in dermatomyositis remains as yet unclear, hampering its present-day utility in early or pre-clinical detection. Thus, identification of high-risk genetic variants, which are generally considered immutable and not subject to reverse causation, may be of use in this context. Several studies have shown a relationship between HLA- and nonHLA genes and increased susceptibility for interstitial lung disease (ILD) in different cohorts, including the HLADRB $1{ }^{*} 03-D Q A 1 * 05-D Q B 1 * 02$ haplotype regardless of myositis subtype or presence of anti-ARS antibodies, in a Caucasian cohort $\left(\mathrm{P}_{\text {corr }}=1 \times 10^{-5}, \mathrm{OR}=5.5\right)(30)$; HLADRB1*04 $(\mathrm{P}=0.01, \mathrm{OR}=2.82)$ and HLA-DRB1*12 $(\mathrm{P}=0.02$, $\mathrm{OR}=2.52$ ), BANK1 (rs3733197; $\mathrm{P}_{\text {corr }}=0.026$ ) and TNFAIP (rs2230926 and rs5029939; $\mathrm{P}_{\text {corr }}<0.05$ for both) in a Chinese Han cohort $(20,25,27)$, and a TNF- $\alpha$ haplotype formed by $-1031 \mathrm{C},-863 \mathrm{~A}$, and $-857 \mathrm{C}(\mathrm{P}=0.05, \mathrm{OR}=2.3)$ in a Japanese cohort (19). Some SNPs have also been found to be protective for ILD; in a cohort of Chinese patients, presence of an ANKRD55 polymorphism (rs7731626) was significantly negatively correlated with both DM-ILD $\left(\mathrm{P}_{\text {corr }}=0.030, \mathrm{OR}=0.65\right)$ and DM/PM-ILD $\left(\mathrm{P}_{\text {corr }}=0.015\right.$, OR $=0.67)(37)$.

In addition to genetic markers, the role of microRNAs (miRNAs), which serve an important epigenetic role in regulating gene expression, have also been explored. MiRNAs are small non-coding RNAs, 20-24 nucleotides long that govern numerous biological processes including skeletal muscle development (38-40). Dysregulation of miRNAs has proven to be pathogenic in many diseases including IIMs. Their stability in serum paraffin-embedded tissue has made them attractive biomarkers (41). To illustrate, one study showed significantly decreased miR-7 expression in IIM/ILD patients compared to those without ILD $(\mathrm{P}<0.05)$, suggesting that circulating miR-7 levels could potentially be used as a biomarker for IIM-ILD (42).

\section{Role in understanding patbogenesis}

Data from omics studies can also be used to elucidate the immunopathogenesis of DM, allowing for discovery of potentially new targets that can guide drug development efforts. Further, differentiating between the key pathogenic players in DM and the other IIMs will allow for a more individualized, nuanced approach to management.

MiRNAs have the potential to be useful in this regard. As mentioned, they are involved in a host of cellular processes and are both tissue-specific and ubiquitous. Identifying the target genes and functional pathways of dysregulated miRNAs can provide unique insights into the mechanistic processes at play in DM. Multiple studies have reported aberrant expression of miRNAs in DM and other IIMs (32,42-47). RNA sequencing on paired serum miRNA and mRNA samples from DM patients showed differential expression of genes involved in interferon signaling and anti-viral response in DM and PM patients compared to controls (44). In one study of a Japanese cohort, levels of hsa-miR-4442 were found to be positively correlated with Skeletal Disease Activity in MITAX (Myositis Intention to Treat Activity Index), with a decrease in levels after treatment with prednisone (48). A study on JDM patients from China showed significant downregulation of mir$193 \mathrm{~b}$ and mir-199b-5p and upregulation of mir-665 in JDM skeletal muscle tissues compared to normal controls (49). Another revealed markedly decreased miR-223 and its predicted target, $\mathrm{PKC} \varepsilon$, in skin biopsies from Gottron's papules of DM and CADM compared to normal and $\mathrm{PsO}$ skin; serum miR-223 concentration was also decreased in $\mathrm{DM} / \mathrm{PM}$ patients, particularly in CADM patients, compared with healthy controls (50). The link between inflammation and muscle wasting was reported in a study that revealed decreased expression of skeletal muscle microRNA-1 (miR-1), miR-133a, and miR-133b in all of the inflammatory myopathy subtypes and miR-206 in DM patients that was significantly inversely correlated with levels of TNF- $\alpha$. Invitro human and mouse models demonstrated that TNF inhibited the expression of these miRNAs and blocked the differentiation of human and mouse myoblasts into myocytes (46). Finally, a relationship between miRNA expression and autoantibody status has been reported, specifically between miR-96-5p upregulation and anti-Jo-1 (44), and miR-150-5p downregulation and antiMDA5 and anti-nuclear matrix protein-2 (NXP2) (51).

Transcriptome profiling has also been performed on DM patients. A microarray study on muscle biopsies from IIM showed significant upregulation of type I IFN-dependent transcripts in IIM muscles; in JDM the top upregulated genes were ISG15 (408-fold), IFIT3 (261-fold), MX1 (99-fold), and IRF7 (37-fold). IFN- $\beta$ (but not IFN- $\alpha$ ) 
transcripts were elevated in DM, JDM, and PM and TLR3 particularly in JDM, supporting central roles of IFN and TLR activation in IIM pathogenesis and suggesting viral contribution to the pathophysiology of IIMs (52). Another microarray-based study on muscle biopsies, this time from pre- and post-IVIG treated DM patients and inclusion body myositis (IBM) patients, showed differential expression of certain genes in the DM patients (all IVIG-responsive) compared to the IBM patients (all IVIG-non responsive); particularly downregulation of interleukin (IL)-22, Kallmann syndrome 1 (KAL-1), ICAM-1, complement C1q, and several structural protein genes, as well as upregulation of CXCL9 and CXCL11. This data provides insight into the mechanism of action of IVIG and emphasizes the differences in DM and IBM pathogenesis (53). RNA sequencing on the PBMCs of DM and PM patients showed significantly more differentially expressed genes in CD8+ compared to CD4+ T cells (176 vs. 2, FDR <0.05), highlighting the central role played by $\mathrm{CD} 8+\mathrm{T}$ cells in both diseases and the uniqueness of the immunopathogenic mechanisms governing DM and PM (31). Lastly, single-cell RNA sequencing on skin biopsies from patients with DM, cutaneous lupus, and healthy controls revealed increased IL-18 expression by keratinocytes in both lesional and nonlesional DM keratinocytes compared to control and lupus biopsies, highlighting a previously undescribed potential role for IL-18 in the pathophysiology of DM skin disease and the value of next-generation sequencing studies in discovering novel mechanistic pathways (35).

Genomic studies can also shed light on the nature of the relationship between environmental and genetic risks factors. One study showed that cigarette smoking in individuals with HLA-DRB1*03:01 allele had the highest odds of developing PM, ILD, anti-synthetase syndrome, and anti-Jo-1 autoantibodies (10). Another showed that the relationship between DM prevalence and geographic latitude was associated with HLA phenotype; HLA alleles significantly associated with anti-Mi-2 (HLADRB $1 * 07: 01$ ) and anti-TIF-1- $\gamma$ autoantibodies (HLA$\left.\mathrm{DQB} 1{ }^{*} 02\right)$ were found to be strongly negatively associated with latitude $(\mathrm{P}<0.001, \mathrm{OR}=0.97$ and $\mathrm{P}<0.001, \mathrm{OR}=0.98$, respectively) (54).

\section{Conclusions}

Despite these promising results, further investigations are necessary before precision medicine tools can be widely delivered from the bench to the bedside. Larger- scale studies on ethnically and geographically diverse cohorts to validate these findings must be performed and computational methods developed to make clinically meaningful sense of the massive, multimodal data sets generated by these studies. In addition, it must be strongly emphasized that while large-scale genotyping and molecular phenotyping in its many exciting, rapidly-evolving forms, represents the shiny new frontier of healthcare delivery, it is not meant to be interpreted in isolation and instead, should always be utilized within the context of the larger clinical picture and other laboratory parameters. Integration of the "big data" mined from these methods with the results of currently available serologic, histopathologic, radiologic, and electrophysiologic tests, and, most importantly, with clinical findings that can only be obtained through good old-fashioned history-taking and physical examination, is the key to elevating the practice of medicine to a more personalized, patient-centric approach that truly optimizes patient outcomes. Thus, while multi-omics may not entirely relieve us of our roles as fortune-tellers, it will at least equip us with a more polished, precise, crystal ball.

\section{Acknowledgments}

Funding: None.

\section{Footnote}

Provenance and Peer Review: This article was commissioned by the Guest Editors (Drs. Richard D. Sontheimer, M. Kari Connolly, David F. Fiorentino, and Victoria P. Werth) for the series "Rheumatologic Skin Disease" published in Annals of Translational Medicine. The article has undergone external peer review.

Conflicts of Interest: Both authors have completed the ICMJE uniform disclosure form (available at http://dx.doi. org/10.21037/atm-20-5319). The series "Rheumatologic Skin Disease" was commissioned by the editorial office without any funding or sponsorship. Dr. ANF reports personal fees from Octagon Therapeutics, outside the submitted work. The authors have no other conflicts of interest to declare.

Ethical Statement: The authors are accountable for all aspects of the work in ensuring that questions related to the accuracy or integrity of any part of the work are appropriately investigated and resolved. 
Open Access Statement: This is an Open Access article distributed in accordance with the Creative Commons Attribution-NonCommercial-NoDerivs 4.0 International License (CC BY-NC-ND 4.0), which permits the noncommercial replication and distribution of the article with the strict proviso that no changes or edits are made and the original work is properly cited (including links to both the formal publication through the relevant DOI and the license). See: https://creativecommons.org/licenses/by-nc-nd/4.0/.

\section{References}

1. Precision Medicine Initiative I The White House [Internet]. [cited 2020 Jan 10]. Available online: https:// obamawhitehouse.archives.gov/precision-medicine

2. FACT SHEET: President Obama's Precision Medicine Initiative I whitehouse.gov [Internet]. [cited 2020 Jan 10]. Available online: https://obamawhitehouse.archives.gov/ the-press-office/2015/01/30/fact-sheet-president-obamas-precision-medicine-initiative

3. Hasin Y, Seldin M, Lusis A. Multi-omics approaches to disease. Genome Biol 2017;18:83.

4. Nguyen ND, Wang D. Multiview learning for understanding functional multiomics. Faeder JR, editor. PLOS Comput Biol 2020;16:e1007677.

5. Qiang JK, Kim WB, Baibergenova A, et al. Risk of Malignancy in Dermatomyositis and Polymyositis. J Cutan Med Surg 2017;21:131-6.

6. Di Rollo D, Abeni D, Tracanna M, et al. Cancer risk in dermatomyositis: a systematic review of the literature. $G$ Ital Dermatol Venereol 2014;149:525-37.

7. Concha JSS, Pena S, Gaffney RG, et al. Developing classification criteria for skin-predominant dermatomyositis: the Delphi process. Br J Dermatol 2020;182:410-7.

8. Miller FW, Lamb JA, Schmidt J, et al. Risk factors and disease mechanisms in myositis. Nat Rev Rheumatol 2018;14:255-68.

9. O'Hanlon TP, Carrick DM, Targoff IN, et al. Immunogenetic risk and protective factors for the idiopathic inflammatory myopathies: distinct HLA-A, -B, -Cw, -DRB1, and -DQA1 allelic profiles distinguish European American patients with different myositis autoantibodies. Medicine (Baltimore) 2006;85:111-27.

10. Schiffenbauer A, Faghihi-Kashani S, O'Hanlon TP, et al. The effect of cigarette smoking on the clinical and serological phenotypes of polymyositis and dermatomyositis. Semin Arthritis Rheum 2018;48:504-12.
11. Bachtiar M, Lee CGL. Genetics of Population Differences in Drug Response. Curr Genet Med Rep 2013;1:162-70.

12. Miller FW, Chen W, O'Hanlon TP, et al. Genomewide association study identifies HLA 8.1 ancestral haplotype alleles as major genetic risk factors for myositis phenotypes. Genes Immun 2015;16:470-80.

13. Rothwell S, Cooper RG, Lundberg IE, et al. Dense genotyping of immune-related loci in idiopathic inflammatory myopathies confirms HLA alleles as the strongest genetic risk factor and suggests different genetic background for major clinical subgroups. Ann Rheum Dis 2016;75:1558-66.

14. O'Hanlon TP, Rider LG, Mamyrova G, et al. HLA polymorphisms in African Americans with idiopathic inflammatory myopathy: allelic profiles distinguish patients with different clinical phenotypes and myositis autoantibodies. Arthritis Rheum 2006;54:3670-81.

15. Rothwell S, Chinoy H, Lamb JA, et al. Focused HLA analysis in Caucasians with myositis identifies significant associations with autoantibody subgroups. Ann Rheum Dis 2019;78:996-1002.

16. O'Hanlon TP, Carrick DM, Arnett FC, et al. Immunogenetic risk and protective factors for the idiopathic inflammatory myopathies: distinct HLA-A, -B, -Cw, -DRB1 and -DQA1 allelic profiles and motifs define clinicopathologic groups in caucasians. Medicine (Baltimore) 2005;84:338-49.

17. Chinoy H, Payne D, Poulton KV, et al. HLA-DPB1 associations differ between DRB $1{ }^{*} 03$ positive anti-Jo- 1 and anti-PM-Scl antibody positive idiopathic inflammatory myopathy. Rheumatology (Oxford) 2009;48:1213-7.

18. Shamim EA, Rider LG, Pandey JP, et al. Differences in idiopathic inflammatory myopathy phenotypes and genotypes between Mesoamerican Mestizos and North American Caucasians: ethnogeographic influences in the genetics and clinical expression of myositis. Arthritis Rheum 2002;46:1885-93.

19. Furuya T, Hakoda M, Tsuchiya N, et al. Immunogenetic features in 120 Japanese patients with idiopathic inflammatory myopathy. J Rheumatol 2004;31:1768-74.

20. Gao X, Han L, Yuan L, et al. HLA class II alleles may influence susceptibility to adult dermatomyositis and polymyositis in a Han Chinese population. BMC Dermatol 2014;14:9.

21. Werth VP, Callen JP, Ang G, et al. Associations of tumor necrosis factor $\alpha$ and HLA polymorphisms with adult dermatomyositis: Implications for a unique pathogenesis. J Invest Dermatol 2002;119:617-20. 
22. Chinoy H, Salway F, John S, et al. Tumour necrosis factor-alpha single nucleotide polymorphisms are not independent of HLA class I in UK Caucasians with adult onset idiopathic inflammatory myopathies. Rheumatology (Oxford) 2007;46:1411-6.

23. Kochi Y, Kamatani Y, Kondo Y, et al. Splicing variant of WDFY4 augments MDA5 signalling and the risk of clinically amyopathic dermatomyositis. Ann Rheum Dis 2018;77:602-11.

24. Sugiura T, Kawaguchi Y, Goto K, et al. Association between a C8orf13-BLK Polymorphism and Polymyositis/ Dermatomyositis in the Japanese Population: An Additive Effect with STAT4 on Disease Susceptibility. Linker RA, editor. PLoS One 2014;9:e90019.

25. Chen S, Wen X, Wang Q, et al. Association between the BANK1 rs3733197 polymorphism and polymyositis/ dermatomyositis in a Chinese Han population. Clin Rheumatol 2019;38:431-6.

26. Chen S, Wen X, Li L, et al. Single nucleotide polymorphisms in the ETS1 gene are associated with idiopathic inflammatory myopathies in a northern Chinese Han population. Sci Rep 2017;7:13128.

27. Chen S, Wang Q, Wu Z, et al. Genetic association study of TNFAIP3, IFIH1, IRF5 polymorphisms with polymyositis/dermatomyositis in Chinese Han population. PLoS One 2014;9:e110044.

28. Wang Q, Chen S, Li Y, et al. Positive association of genetic variations in the phospholipase C-like 1 gene with dermatomyositis in Chinese Han. Immunol Res 2016;64:204-12.

29. Sandoval-García F, Petri M, Saavedra M, et al. The ACTN3 R577X polymorphism is associated with inflammatory myopathies in a Mexican population. Scand J Rheumatol 2012;41:396-400.

30. Chinoy H, Salway F, Fertig N, et al. In adult onset myositis, the presence of interstitial lung disease and myositis specific/associated antibodies are governed by HLA class II haplotype, rather than by myositis subtype. Arthritis Res Ther 2006;8:R13.

31. Houtman M, Ekholm L, Hesselberg E, et al. T-cell transcriptomics from peripheral blood highlights differences between polymyositis and dermatomyositis patients. Arthritis Res Ther 2018;20:188.

32. Gao S, Zhang H, Zuo X, et al. Integrated comparison of the miRNAome and mRNAome in muscles of dermatomyositis and polymyositis reveals common and specific miRNA-mRNAs. Epigenomics 2019;11:23-33.

33. Karasawa R, Jarvis JN. Using proteomic and genomic methods to understand JDM. Nihon Rinsho Meneki Gakkai Kaishi 2015;38:150-6.

34. Tezak Z, Hoffman EP, Lutz JL, et al. Gene expression profiling in DQA ${ }^{*} 0501$ + children with untreated dermatomyositis: a novel model of pathogenesis. J Immunol 2002;168:4154-63.

35. Tsoi LC, Gharaee-Kermani M, Berthier CC, et al. IL-18containing five-gene signature distinguishes histologically identical dermatomyositis and lupus erythematosus skin lesions. JCI Insight 2020;5:e139558.

36. Stuhlmüller B, Schneider U, González-González JB, et al. Disease specific autoantibodies in idiopathic inflammatory myopathies. Front Neurol 2019;10:438.

37. Li L, Chen S, Wen X, et al. Positive Association between ANKRD55 Polymorphism 7731626 and Dermatomyositis/ Polymyositis with Interstitial Lung Disease in Chinese Han Population. Biomed Res Int 2017;2017:2905987.

38. Chen W, Liu D, Li QZ, et al. The function of ncRNAs in rheumatic diseases. Epigenomics 2019;11:821-33.

39. Seo J, Jin D, Choi CH, et al. Integration of MicroRNA, mRNA, and protein expression data for the identification of cancer-related MicroRNAs. PLoS One 2017;12:e0168412.

40. Lam NT, Gartz M, Thomas L, et al. Influence of microRNAs and exosomes in muscle health and diseases. J Muscle Res Cell Motil 2020;41:269-84.

41. Hydbring $P$, Badalian-Very G. Clinical applications of microRNAs. F1000Research 2013;2:136.

42. Yu L, Li J, Chen Y, et al. hsa-miR-7 Is a Potential Biomarker for Idiopathic Inflammatory Myopathies with Interstitial Lung Disease in Humans. Ann Clin Lab Sci 2018;48:764-9.

43. Parkes JE, Day PJ, Chinoy H, et al. The role of microRNAs in the idiopathic inflammatory myopathies. Curr Opin Rheumatol 2015;27:608-15.

44. Parkes JE, Thoma A, Lightfoot AP, et al. MicroRNA and mRNA profiling in the idiopathic inflammatory myopathies. BMC Rheumatol 2020;4:25.

45. Jiang T, Huang Y, Liu H, et al. Reduced miR-146a Promotes REG3A Expression and Macrophage Migration in Polymyositis and Dermatomyositis. Front Immunol 2020;11:37.

46. Georgantas RW, Streicher K, Greenberg SA, et al. Inhibition of myogenic microRNAs 1, 133, and 206 by inflammatory cytokines links inflammation and muscle degeneration in adult inflammatory myopathies. Arthritis Rheumatol 2014;66:1022-33.

47. Misunova M, Salinas-Riester G, Luthin S, et al. Microarray 
analysis of circulating micro RNAs in the serum of patients with polymyositis and dermatomyositis reveals a distinct disease expression profile and is associated with disease activity. Clin Exp Rheumatol 2016;34:17-24.

48. Hirai T, Ikeda K, Tsushima H, et al. Circulating plasma microRNA profiling in patients with polymyositis/ dermatomyositis before and after treatment: miRNA may be associated with polymyositis/dermatomyositis. Inflamm Regen 2018;38:1.

49. Qiu CC, Su QS, Zhu SY, et al. Identification of Potential Biomarkers and Biological Pathways in Juvenile Dermatomyositis Based on miRNA-mRNA Network. Biomed Res Int 2019;2019:7814287.

50. Inoue K, Jinnin M, Yamane K, et al. Down-regulation of miR-223 contributes to the formation of Gottron's papules in dermatomyositis via the induction of PKC $\varepsilon$. Eur J Dermatol 2013;23:160-7.

Cite this article as: Castillo RL, Femia AN. Polishing the crystal ball: mining multi-omics data in dermatomyositis. Ann Transl Med 2021;9(5):435. doi: 10.21037/atm-20-5319
51. Ye L, Zuo Y, Yang H, et al. Specific Autoantibodies and Clinical Phenotypes Correlate with the Aberrant Expression of Immune-Related MicroRNAs in Dermatomyositis. J Immunol Res 2019;2019:2927061.

52. Cappelletti C, Baggi F, Zolezzi F, et al. Type I interferon and Toll-like receptor expression characterizes inflammatory myopathies. Neurology 2011;76:2079-88.

53. Raju R, Dalakas MC. Gene expression profile in the muscles of patients with inflammatory myopathies: effect of therapy with IVIg and biological validation of clinically relevant genes. Brain 2005;128:1887-96.

54. Parkes JE, Rothwell S, Oldroyd A, et al. Genetic background may contribute to the latitude-dependent prevalence of dermatomyositis and anti-TIF1- $\gamma$ autoantibodies in adult patients with myositis. Arthritis Res Ther 2018;20:117. 